\title{
Utilização de Objetos de Aprendizagem Abertos SCORM para dar suporte à Avaliação Formativa
}

\author{
Renato Dutra \\ Pós-Graduação em Informática na Educação \\ Centro Interdisciplinar de Novas Tecnologias na Educação \\ Universidade Federal do Rio Grande do Sul \\ Escola de Engenharia e Tecnologia \\ Universidade Anhembi Morumbi \\ rlsdutra@anhembi.edu \\ Liliana Passerino \\ Pós-Graduação em Informatica na Educação \\ Centro Interdisciplinar de Novas Tecnologias na Educação \\ Universidade Federal do Rio Grande do Sul \\ liliana@cinted.ufrgs.br
}

\author{
Liane Tarouco \\ Pós-Graduação em Informática na Educação \\ Centro Interdisciplinar de Novas Tecnologias na Educação \\ Universidade Federal do Rio Grande do Sul \\ liane@penta.ufrgs.br
}

Resumo A avaliação formativa, apesar de ter sido discutida por diversos autores nas últimas décadas, ainda é pouco utilizada, tanto nos cursos presenciais como a distância. Somente nos últimos anos, com a preocupação maior com o processo de ensino-aprendizagem e com o crescimento dos cursos a distância, essa abordagem de avaliação começou a ganhar mais importância. Na Educação a Distância, a avaliação formativa tem se mostrado uma excelente alternativa, por propiciar aos professores um acompanhamento mais próximo e mais individualizado de seus alunos e por permitir a regulação do processo de ensino-aprendizagem. Independente do processo avaliativo, o desenvolvimento de conteúdos digitais educacionais na forma de objetos de aprendizagem tem sido uma solução crescente na EAD e para tanto, modelos de portabilidade e interoperabilidade, como o SCORM, proporcionaram uma grande evolução em termos de reusabilidade, portabilidade e interoperabilidade. Dessa forma, o desenvolvimento de objetos de aprendizagem deveria levar em consideração o processo de avaliação formativa. Com base nessas constatações, a presente pesquisa buscou identificar quais critérios deveriam ser levados em consideração para o encapsulamento e a utilização de Objetos de Aprendizagem SCORM em Ambientes Virtuais de Aprendizagem, a fim de apoiar o processo de avaliação formativa.

Palavras-Chave: Avaliação formativa. SCORM. Objeto de Aprendizagem.

\begin{abstract}
Formative evaluation is still little employed in both face-to-face and distance learning courses, although it has been discussed by various authors in the last few decades. Only recently has this evaluation approach started to gain importance, with a mounting concern over the teaching-learning process, and an increase in distance learning courses. In distance learning, formative evaluation has proved to be an excellent alternative, since it provides teachers a closer and more individualized observation of their students allowing to adjust the teachinglearning process. Regardless of the evaluation process, the development of educational digital contents in the form of learning objects has increasingly presented itself as a solution in distance learning. For this purpose, portability and interoperability models such as SCORM have provided a great advancement in terms of reusability, portability and interoperability. Therefore, the development of learning objects should take into account the formative evaluation process. Based on such findings, the present research aims to identify which criteria should be considered in the process of encapsulate and use of SCORM Learning Objects in Virtual Learning Environments, in order to support the formative evaluation process.
\end{abstract}

Keywords: Formative evaluation. SCORM. Learning objects. 


\section{Introdução}

Apesar da avaliação formativa ter sido inicialmente proposta por Scriven há mais de 30 anos [14], ela ainda é pouco utilizada nos espaços educativos tendo ganho nos últimos anos mais destaque. Na Educação a Distância (EAD), a avaliação formativa tem se mostrado uma excelente alternativa, por propiciar aos professores um acompanhamento mais próximo de seus alunos e por permitir que professores e alunos regulem o processo de ensinoaprendizagem.

Por outro lado, independente do processo avaliativo, o desenvolvimento de conteúdos digitais educacionais na forma de objetos de aprendizagem tem sido uma solução crescente na EAD. Para tanto, padrões de interoperabilidade, como o SCORM, proporcionaram uma grande evolução em termos de reusabilidade, portabilidade e interoperabilidade [19], permitindo que o ambiente acadêmico possa facilmente intercambiar objetos de aprendizagem, independente da plataforma de hardware e software. Dessa forma, o desenvolvimento de objetos de aprendizagem SCORM, bastante utilizados na EAD, deveria levar em consideração o processo de avaliação formativa.

O presente artigo apresenta uma sugestão de utilização de objetos de aprendizagem SCORM, bem como os elementos de dados do modelo SCORM, como forma de apoio para a avaliação formativa.

\section{Avaliação da Aprendizagem e Ava- liação Formativa}

A denominação "Avaliação da Aprendizagem" na educação é atribuída a Ralph Tyler na década de 30 [6]. Contribuindo com os pensamentos de Tyler, Michael Scriven em 1963, complementou diferenciando o papel formativo e somativo da avaliação da aprendizagem, cunhando pela primeira vez o termo "avaliação formativa" [14]

Quando escreveu sobre o tema, o autor se referia à avaliação formativa no sentido de melhorar o curso durante seu andamento, como um instrumento no qual o professor estaria altamente engajado na melhoria de seu material. Evoluindo o conceito, Bloom e seus colaboradores [2] ampliaram a visão de Scriven para além do material e do curso, focando mais no processo de ensino/aprendizagem e propondo alguns instrumentos para avaliação formativa.

Apesar de sua primeira proposição ter ocorrido há quase 50 anos, a aplicação da avaliação formativa tem sido mais significativa nas últimas décadas, a partir das experiências de ciclos de aprendizagem, na preocupação de respeitar as diferenças individuais dos alunos e de regular melhor o processo de ensino-aprendizagem [3]. Mesmo assim, ainda hoje a avaliação formativa é pouco utilizada.

Embora existam diferenças nas concepções de avaliação formativa de diversos autores, a partir de Bloom elas têm em comum a mesma essência. Todos os autores sugerem que a avaliação formativa é realizada durante o processo de ensino/aprendizagem, que ela é contínua, que ela não deve ser característica classificatória ou certificadora e que se baseia fortemente no feedback, tanto para o professor como para o aluno [2] [17] [10] [5].

Hadji [5] acrescenta que a avaliação formativa apresenta três características principais: ela é informativa, pois informa os dois atores do processo de ensino/aprendizagem; ela informa ao professor, que será informado dos efeitos reais de suas ações, podendo regular sua ação pedagógica, e ao aprendiz, que terá oportunidade de tomar consciência de suas dificuldades e, possivelmente, reconhecer e corrigir seus próprios erros; ela é reguladora, pois permite ao professor e ao aluno corrigir suas ações, modificando-as, se necessário, a fim de obter melhores resultados.

Por suas características, a avaliação formativa apresenta-se como um instrumento apropriado para acompanhar a evolução e a performance do aluno na Educação a Distância [18], visto que qualquer dificuldade encontrada pelos aprendizes pode ser orientada antes que se torne um grande obstáculo à aprendizagem.

Ademais, o que pode ser observado ao longo do tempo é que a avaliação formativa tem se mostrado de vital importância para apoiar a percepção do comportamento dos aprendizes a distância, pois proporciona uma orientação mais adequada à atividade em andamento, em um contexto em que o professor não tem contato presencial com o aluno [9].

A utilização de Ambientes Virtuais de Aprendizagem (AVA) na avaliação formativa oferece uma riqueza de informações registradas no ambiente, cabendo ao professor transformar esse conjunto de informações em subsídios para uma avaliação [7]. Entretanto, é importante haver um suporte adequado ao processo de avaliação formativa on-line, pois esse modelo de avaliação pode gerar uma grande sobrecarga para os professores [9] [12]. Essa sobrecarga normalmente se dá pela necessidade de análise de informações sobre a participação do aluno, dispersas no AVA.

Diversos estudos e trabalhos já foram realizados no sentido de apoiar a avaliação formativa na EAD. Esses estudos incluem a utilização dos AVAs, de seus mecanismos de rastreamento e de recursos de Inteligência artificial tais como Raciocínio Baseado em Casos e Sis- 
temas Multiagentes, demonstrados pelos trabalhos de Weirich e seus colaboradores [15], Otsuka [9], Rodrigues [13], Prata [12], Pimentel e seus colaboradores [11], Piva Jr e seus colaboradores [23], entre outros.

Em todos esses trabalhos observa-se que um mecanismo importante para o processo de avaliação é o rastreamento e acompanhamento das atividades e ações dos alunos dentro do AVA. Além disso, outra informação utilizada é a oriunda do registro de respostas dos exercícios que foram realizados dentro do próprio AVA. Entretanto, o que se verifica é que nenhuma das soluções pode ser facilmente portada, por terem sido desenvolvidas especificamente para um determinado AVA. Nesse sentido, é necessária a busca de soluções que permitam uma fácil portabilidade e reusabilidade dos recursos de avaliação, tendo em vista a grande diversidade de AVAs utilizados por instituições de ensino no Brasil e no mundo. Uma alternativa é a utilização de Objetos de Aprendizagem SCORM como mecanismo de avaliação formativa.

\section{Objetos de Aprendizagem SCORM}

SCORM (Sharable Content Object Reference Model) é um conjunto unificado de recomendações que sugere quais serviços são necessários para disponibilizar unidades de aprendizagem (cursos, lições, aulas, etc.) on-line via Web, como essas unidades podem ser 'empacotadas', quais padrões existentes devem ser aplicados, e também a maneira como esses padrões devem ser utilizados [1].
O modelo SCORM é formado por um conjunto de especificações publicadas na forma de livros. Nesses livros estão compiladas recomendações acerca da organização dos conteúdos, como esses conteúdos são estruturados e como será a navegação neles. Entretanto, sua principal característica é possibilitar o rastreamento e acompanhamento das ações do aluno dentro do AVA, no que diz respeito à interação do aluno com o conteúdo (objeto de aprendizagem) propriamente dito. Essas informações estão descritas no livro 'Ambiente de Execução', que define um Mecanismo de Execução comum para a comunicação entre o AVA e os objetos de aprendizagem SCORM (SCO), bem como um modelo de dados comum para padronizar e apoiar essa comunicação, possibilitando o rastreamento das atividades do aluno. (quadro 1)

\subsection{Utilização do SCORM na Avaliação Formativa}

Como mencionado anteriormente, o SCORM, em sua especificação do Ambiente de Execução, estipula um modelo de dados que contém elementos que podem ser intercambiados entre o AVA e os objetos de aprendizagem SCORM (SCO). Alguns desses elementos são coletados automaticamente pela simples inicialização e finalização do SCO, outros precisam ser programados para tal fim dentro dos SCO, através de chamadas padronizadas na linguagem JavaScript.

\begin{tabular}{|l|l|l|}
\hline $\begin{array}{l}\text { Elemento do } \\
\text { modelo de dados }\end{array}$ & Notação do elemento & Descrição \\
\hline $\begin{array}{l}\text { Comentários do } \\
\text { aprendiz }\end{array}$ & cmi.comments_from_learner & Contém comentários e textos do aprendiz sobre o SCO. \\
\hline $\begin{array}{l}\text { Comentários do } \\
\text { AVA }\end{array}$ & cmi.comments_from_lms & $\begin{array}{l}\text { Contém comentários e anotações do SCO com o objetivo de serem mostra- } \\
\text { das para os aprendizes. }\end{array}$ \\
\hline Interações & cmi.interactions & $\begin{array}{l}\text { Define informações pertinentes às interações (testes, exercícios, etc.) com o } \\
\text { propósito de medição ou avaliação. }\end{array}$ \\
\hline Objetivos & cmi.objectives & $\begin{array}{l}\text { Especifica os objetivos de aprendizagem ou performance associados com } \\
\text { cada SCO. }\end{array}$ \\
\hline Progresso medido & cmi.progress_measure & Mede o progresso que o aluno está tendo no SCO. \\
\hline Escore & cmi.score & Identifica a pontuação do aluno para um SCO. \\
\hline Status de sucesso & cmi.success_status & Indica quando o aluno finalizou com sucesso o SCO. \\
\hline Tempo total & cmi.total_time & $\begin{array}{l}\text { Identifica o tempo total acumulado de todas as sessões na tentativa corrente } \\
\text { do aluno. }\end{array}$ \\
\hline
\end{tabular}

Quadro 1: Conjunto de Elementos importantes para a Avaliação Formativa

Esses elementos podem ser registrados de forma correta e observados através dos relatórios de rastreamento dos AVAs, podendo ser utilizados para a avaliação formativa. O quadro 1 indica quais elementos do SCORM poderiam ser utilizados para esse fim.

Detalhando um pouco mais os elementos sugeridos para apoiar a avaliação formativa, os elementos de co- 
mentários do aluno (cmi.comments_from_learner) podem ser utilizados para registro das impressões sobre a experiência de aprendizagem no SCO, para registrar anotações de um diário de bordo e para armazenar respostas as questões dissertativas, entre outras coisas. Já os comentários do AVA (cmi.comments_from_lms) contêm informações que devem ser vistas pelos alunos quando estes estiverem navegando nos SCOs, desde que o SCO contenha tais elementos registrados.

Já o conjunto de elementos das interações (cmi.interactions) é uma forma de registrar as respostas dos alunos em exercícios. As interações são respostas para exercícios ou atividades individuais que se queiram registrar em um SCO. Para o AVA, a única obrigação é registrar esses dados quando solicitados, não tendo que proceder com nenhum comportamento específico. São excelentes instrumentos para a avaliação formativa por permitirem o registro da resposta exata do aluno em tais exercícios, possibilitando a análise posterior do professor. Com os diversos tipos de interação permitidos, é possível inserir tais interações para avaliar o nível de dificuldade dos alunos.

Outro conjunto de elementos úteis do SCORM são os objetivos educacionais (cmi.objectives), em que para cada SCO podem ser identificados um ou mais objetivos. Os objetivos são tratados como agrupamentos do conjunto de elementos de dados dos objetivos para um determinado $\mathrm{SCO}$, os quais podem ser usados para rastrear a aprendizagem do aluno.

O elemento do modelo de dados intitulado 'Score' (cmi.score) é utilizado para registrar a pontuação ou nota do aluno no SCO. Muitas vezes, no fim de cada SCO, uma avaliação objetiva pode ser introduzida para avaliar o desempenho do aluno no SCO, registrando sua pontuação. Apesar de seu aparente enfoque somativo, a pontuação pode ser mais um dado observável no processo de avaliação formativa, desde que utilizado com outros exercícios e analisado no conjunto de elementos registrados para um SCO.

O tempo de Sessão (cmi.session_time) é um dos elementos mais utilizados nos relatórios de atividades SCORM e demonstra o total de tempo que o aluno gastou na última sessão para determinado SCO, sendo que o tempo total (cmi.total_time) é a soma de todos os tempos de sessão registrados para o SCO. Esses elementos são utilizados para acompanhar o tempo gasto pelos alunos em todas as suas sessões para uma determinada tentativa [1]. Através dele, o avaliador pode verificar se o aluno está levando mais tempo para navegar por um determinado SCO do que outros alunos, o que pode indicar possíveis dificuldades do aluno naquela parte do conteúdo.

A medida de progresso (cmi.progress_measure) é o elemento utilizado para aferir o progresso do aluno na execução do SCO. Pode ser definido como 'not attempted', para quando ainda não foi executado; 'completed', para quando foi executado até o final; ou 'incomplete', quando foi executado sem chegar ao final. $\mathrm{O}$ mecanismo de definição do valor desse elemento é determinado pelo desenvolvedor e está implícito no algoritmo do SCO. Através desse elemento, é possível que o avaliador verifique se o aluno conseguiu chegar ou não ao final do SCO. Em conjunto com os elementos tempo total e registro do número de tentativas, o avaliador pode inferir se o aluno está tendo dificuldades nos conteúdos estudados.

O último elemento recomendado para o apóia à avaliação formativa é o status do sucesso (cmi.success_status), ele indica quando o aluno atingiu com êxito o final do SCO. O SCORM não define como esse sucesso deve ser determinado, mas o SCO pode indicar esse status baseando-se no percentual das interações realizadas, objetivos alcançados, pontuação atingida em uma interação, etc. Como o critério de sucesso é determinado pelo desenvolvedor do SCO, ele pode ser utilizado de acordo com as diretrizes do avaliador para facilitar o acompanhamento do aluno na avaliação formativa [1].

Todos esses elementos, para serem corretamente registrados, demandam a intervenção do desenvolvedor do objeto de aprendizagem ( $\mathrm{SCO}$ ), incluindo comandos que invoquem seu correto registro, sendo muitas vezes necessária a readequação e a adição de comandos em objetos de aprendizagem existentes. Além disso, é necessário o empacotamento desses objetos de aprendizagem já modificados em unidades de aprendizagem SCORM, por meio de ferramentas de empacotamento, preferencialmente de software livre.

\subsection{SCORM para criar Objetos de Aprendi- zagem Abertos}

A necessidade de interoperabilidade dos conteúdos digitais educacionais visando a avaliação formativa e o acompanhamento das ações do aluno remete à utilização de OA em modelos e padrões institucionalizados como o SCORM.

Entretanto, para que os OA possam ser reutilizáveis e intercambiáveis entre diversas instituições, é importante que sejam feitos utilizando licenças abertas, seguindo o mesmo modelo que consagrou as experiências de software livre. Esses OA disponibilizados sob licenças abertas são o que convencionamos chamar de Objetos de Aprendizagem Abertos (OAA).

As licenças abertas para conteúdos, bem como todo um movimento para o desenvolvimento de conteúdos digitais dedicados a fins educacionais, foram inspiradas no movimento e nas licenças de software livre. O amadu- 
recimento dessas licenças iniciou-se com a Open Publication License [19], culminando com o surgimento da licença Creative Commons [19] [22], que permite ampla liberdade na definição de quais direitos o autor original está abrindo mão. Paralelamente ao surgimento da Creative Commons, o MIT criou sua iniciativa de Open Courseware [21], uma forma de disponibilizar para a comunidade, sem custo de utilização, materiais digitais utilizados como apoio em sala de aula.

Com licenças que permitiam a livre cópia, distribuição e modificação de conteúdos e com o incentivo da disponibilização de material educacional de uma universidade americana de primeiro nível, a idéia de conteúdos abertos ganhou mais força e mais adeptos ao redor do mundo.

Paralelamente a esse movimento, foram surgindo repositórios e ferramentas de catalogação para que esses objetos de aprendizagem estivessem facilmente acessíveis, ou para que a combinação desses objetos em diferentes unidades de aprendizagem fosse bem-sucedida. Embora muitos desses repositórios já contenham informações genéricas sobre a licença utilizada, é importante que eles prevejam informações detalhadas do tipo de licença utilizado e forneçam formas de acessar os códigos-fonte dos objetos de aprendizagem, visto que, por serem abertos, esses objetos devem poder ser modificados e reutilizados.

Acreditamos que esses recursos que combinam os OAA sob licenças Creative Commons [22], contendo conteúdos e exercícios que visem o acompanhamento do aluno, devidamente empacotados com seus arquivos-fonte no modelo SCORM, podem efetivamente apoiar a avaliação formativa de maneira uniformizada. Outrossim, entendemos que essa solução pode garantir às instituições de ensino independência de plataforma, bem como uma verdadeira reusabilidade ou interoperabilidade.

\section{Metodologia da Pesquisa}

A questão que norteou este trabalho foi a identificação de critérios e elementos para direcionar o encapsulamento de objetos de aprendizagem em AVAs, a fim de apoiar a avaliação formativa e, ao mesmo tempo, a garantia de portabilidade e rastreabilidade desses objetos.

A presente pesquisa que aliou a avaliação formativa dos objetos de aprendizagem e a reusabilidade destes, pode ser caracterizada como exploratória, visto que objetivou proporcionar maior familiaridade com o problema a fim de torná-lo mais explícito [4].

Para responder ao problema proposto e aos objetivos gerais e específicos, decidiu-se por executar a pesquisa em quatro fases distintas: na primeira fase foi necessário estudar detalhadamente todo o processo de avaliação formativa, os diversos padrões de interoperabilidade, os mecanismos do ambiente de execução do SCORM, e o conceito de conteúdos abertos, conceitos estes demonstrados no inicio deste artigo; com base nisso, em uma segunda fase foi proposto um conjunto de recomendações para o encapsulamento de objetos de aprendizagem abertos encapsulados em SCORM, bem como a condução da avaliação formativa baseada em tais objetos de aprendizagem; uma vez definido esse modelo, foi necessário validá-lo, o que foi realizado na terceira fase através de um estudo de caso; na quarta fase, como resultado da validação, foi criado um CD com tutoriais e indicações.

Especificamente na terceira fase, partindo das características da avaliação formativa, sua aplicação na EAD e os elementos do modelo de dados do SCORM estudados e elencados nas primeira e segunda fases, buscou-se uma validação para verificar se o SCORM poderia ser utilizado de forma eficaz para a avaliação formativa. Para isso, optou-se por uma pesquisa qualitativa através de um estudo de caso.

$\mathrm{O}$ estudo de caso foi realizado em uma turma piloto do curso de tutores do Programa Nacional Escola Nacional de Gestores do MEC. O curso foi realizado nos meses de fevereiro e março de 2008, utilizando o AVA Moodle e sendo coordenado pela equipe do Centro Interdisciplinar de Novas Tecnologias na Educação (CINTED) da UFRGS.

Com relação aos aspectos metodológicos, apesar de ser uma pesquisa de cunho qualitativo, considerou-se também alguns métodos quantitativos, como a utilização de um pré-experimento, caracterizando a pesquisa como metodologia "mista" [8]. O estudo de caso utilizou a abordagem de projeto de caso único [16], visto que um curso específico para tutores, com a possibilidade de sofrer algumas intervenções a fim de utilizar exercícios SCORM, delineou-se como uma oportunidade única de pesquisa.

Foi utilizado, no estudo de caso, o método da observação direta do tipo estruturada [8], cujo objetivo foi avaliar a qualidade e a utilidade dos dados de rastreamento registrados no Moodle. No entanto, somente a observação não seria suficiente para uma validação, mesmo se tratando de uma pesquisa exploratória. Buscou-se, então, nos procedimentos experimentais de natureza mais quantitativa, complementar a pesquisa com a aplicação de uma intervenção no curso, acompanhada de um questionário no final, para permitir a validação sem comprometer a presente pesquisa, caracterizando essa etapa como um pré-experimento.

O estudo de caso foi organizado em três etapas consecutivas. A primeira visou à inclusão de Objetos de A- 
prendizagem SCORM (exercícios) no curso-piloto, desenvolvidos através da ferramenta Hot Potatoes, sendo criados 46 (quarenta e seis) exercícios SCORM distribuídos entre os 10 tópicos do curso, para possibilitar o acompanhamento do rastreamento registrado. A segunda etapa correspondeu à criação de um tópico específico no curso para a aplicação do questionário que visava coletar as impressões dos alunos e do tutor com relação à avaliação formativa. Como muitos alunos não tinham conhecimentos de Avaliação Formativa nem dos mecanismos do SCORM, foi criado também um $11^{\circ}$ tópico no curso abordando e explicando o processo de avaliação formativa por meio do SCORM. A terceira etapa correspondeu à liberação do tópico e aplicação do questionário para os alunos e para o tutor, bem como a aplicação de um questionário com questões abertas especificamente para o tutor.

\section{Análise e Interpretação dos Dados}

Os dados coletados no estudo de caso foram obtidos por meio de uma observação estruturada no AVA Moodle e através de um pré-experimento com a coleta de respostas do questionário aplicado aos alunos e ao tutor do curso. A ideia por trás da observação foi, por um lado, observar o comportamento dos alunos em relação às atividades SCORM propostas e, por outro, verificar a forma e a qualidade dos dados registrados por tais atividades. Já o pré-experimento teve o objetivo de coletar as impressões que os sujeitos (os alunos futuros tutores e o tutor do curso) tiveram das atividades SCORM, dos dados coletados por meio de tais atividades e da viabilidade de utilização desses dados para a avaliação formativa.

\begin{tabular}{|c|c|c|c|c|c|}
\hline Questões & $\begin{array}{l}\text { Discordo } \\
\text { totalmente }\end{array}$ & Discordo & $\begin{array}{l}\text { N.concordo } \\
\text { n.discordo }\end{array}$ & Concordo & $\begin{array}{l}\text { Concordo } \\
\text { totalmente }\end{array}$ \\
\hline $\begin{array}{l}\text { Q1 - As informações contidas nos relatórios } \\
\text { possibilitam saber se os alunos atingiram os objetivos } \\
\text { propostos para cada tópico. }\end{array}$ & 0 & 0 & 1 & 5 & 5 \\
\hline $\begin{array}{l}\text { Q2 - As informações contidas nos relatórios } \\
\text { possibilitam saber qual o nível de progresso dos } \\
\text { alunos. }\end{array}$ & 0 & 0 & 0 & 6 & 5 \\
\hline $\begin{array}{l}\text { Q3 - As informações contidas nos relatórios fornecem } \\
\text { indícios de quais são as dificuldades dos alunos. }\end{array}$ & 0 & 0 & 0 & 7 & 4 \\
\hline $\begin{array}{l}\text { Q4-O texto da atividade de auto-avaliação subjetiva } \\
\text { de cada tópico fornece indícios de quais são as } \\
\text { dificuldades de entendimento dos alunos sobre o } \\
\text { tópico. }\end{array}$ & 0 & 0 & 0 & 3 & 8 \\
\hline $\begin{array}{l}\text { Q5 - O texto da atividade de auto-avaliação subjetiva } \\
\text { de cada tópico possibilita avaliar se os alunos atingiu } \\
\text { os objetivos de cada tópico. }\end{array}$ & 0 & 0 & 0 & 7 & 4 \\
\hline $\begin{array}{l}\text { Q6 - O conjunto de informações das atividades } \\
\text { SCORM pode ser útil para o aluno acompanhar seu } \\
\text { progresso e dificuldades, auxiliando-o a dosar seu } \\
\text { ritmo e esforço nos estudos e nas atividades do curso }\end{array}$ & 1 & 0 & 0 & 6 & 4 \\
\hline $\begin{array}{l}\text { Q7 - O conjunto de informações das atividades } \\
\text { SCORM são úteis para o processo de avaliação } \\
\text { formativa }\end{array}$ & 0 & 0 & 0 & 3 & 8 \\
\hline TOTAL & 1 & 0 & 1 & 37 & 38 \\
\hline
\end{tabular}

Tabela 1: Níveis de concordância acerca dos recursos SCORM para a Avaliação Formativa

\subsection{Resultados da Observação}

A observação direta estruturada ocorreu por meio do relatório das atividades SCORM no Moodle. O processo de observação por meio das atividades SCORM realizouse com a análise diária das informações contidas nos relatórios, visando o acompanhamento de todas as 46 (quarenta e seis) atividades SCORM do curso.

\begin{tabular}{|l|r|}
\hline Total de Tentativas & $\mathbf{7 3 7}$ \\
\hline Tempo total nas atividades & $\mathbf{2 : 2 0 : 4 5}$ \\
\hline Média de alunos participantes nas atividades & $\mathbf{1 5}$ \\
\hline Média de Tentativas & $\mathbf{1 , 1 0}$ \\
\hline Tempo médio em cada atividade & $\mathbf{0 : 0 3 : 0 4}$ \\
\hline Pontuação Média nas atividades & $\mathbf{8 3 , 0 9 9 4}$ \\
\hline
\end{tabular}

Quadro 2: Resumo dos dados do Tracking 
O quadro 2 resume os dados coletados através da observação. Conforme pode ser observado, houve 15 alunos em média em cada atividade. Das primeiras atividades participaram mais de 30 (trinta) alunos, enquanto pouco mais que $10(\mathrm{dez})$ participaram das atividades finais. $\mathrm{O}$ tempo médio dos alunos ficou em torno de $03 \mathrm{~m} 04 \mathrm{~s}$, variando muito de acordo com cada atividade. Já a pontuação dos alunos obteve média elevada em todas as atividades SCORM, com um valor médio de 83 de 100 possíveis, sendo que a distribuição da média das pontuações nas atividades foi bastante homogênea.

Ao final da compilação dos dados e da análise do rastreamento dos alunos, contido nos relatórios de atividades SCORM, constatamos que, com relação à qualidade dos registros de rastreamento, eles apresentam todas as informações das atividades SCORM e possibilitam o acompa- nhamento pelo professor, permitindo-lhe regular as atividades do curso e remediar os alunos com maiores dificuldades.

\subsection{Resultados do Pré-experimento}

O pré-experimento foi aplicado a dois grupos de sujeitos: os alunos que finalizaram o curso de tutores e o tutor do curso. Os alunos eram 55 (cinqüenta e cinco) no início do curso, sendo que somente 20 (vinte) finalizaram. Desses 20 , somente 11 (onze) responderam ao questionário proposto.

A análise das respostas dos sujeitos alunos do curso de tutores, de característica mais quantitativa, foi baseada nas respostas dos 11 (onze) que responderam ao questionário sobre avaliação formativa e SCORM.

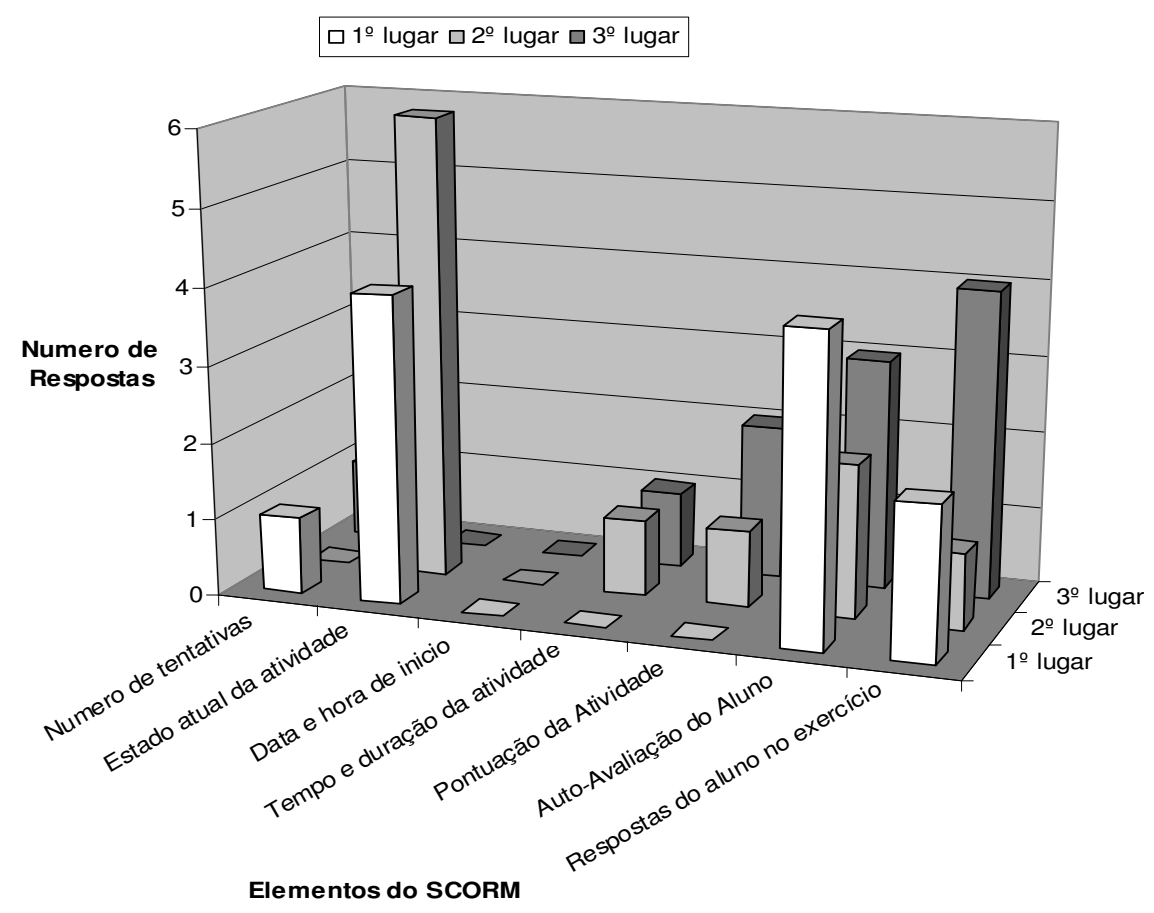

Figura 1: Os elementos do SCORM destacados nos resultados da pesquisa

Analisando os dados da tabela 1, observamos que, de um modo geral, os sujeitos que responderam concordam que o SCORM e seus dados de rastreamento podem ser utilizados tanto por alunos como por professores no processo de avaliação formativa. Quando os sujeitos foram solicitados a ordenar em grau de importância os elementos do SCORM demonstrados no relatório de atividades do Moodle, verificamos que, segundo eles, os elementos mais importantes são o 'estado atual da atividade' (completado, incompleto ou não iniciado), a auto-avaliação do aluno (resposta dissertativa gravada no 'cmi.comments_from_learner') e as respostas dos alunos nos exercícios. Isso pode ser visualizado na figura 1 , em que destacamos as escolhas dos sujeitos até o $3^{\circ}$ lugar.

A última parte do questionário, contendo duas questões abertas, foi incluída com a intenção de captar informações de caráter mais qualitativo não identificadas nas questões anteriores. Observando os comentários dos alunos quando perguntados na primeira questão se acrescentariam alguma informação no relatório de atividades SCORM para apoiar a avaliação formativa, grande parte afirmou que os dados fornecidos eram suficientes. 
Na segunda questão aberta, em que os sujeitos eram convidados a dar suas considerações finais sobre SCORM e a avaliação formativa, todos, sem exceção, teceram comentários favoráveis ao uso da avaliação formativa e do SCORM.

Por último, queríamos avaliar junto aos alunos quais outras atividades do Moodle, além do SCORM, poderiam apoiar o processo de avaliação formativa. Para tal informação, foi utilizada uma questão de múltipla escolha. Quando analisamos as respostas dos sujeitos nessa questão, verificamos que a atividade de fórum foi a considerada mais importante, com 8 (oito) respostas, seguida pelos diários, com 7 (sete) respostas, e chats, com 5 (cinco) respostas. Entre todas as atividades do Moodle, estas são as mais focadas na interação e na comunicação entre os participantes do curso, principalmente em um curso a distância sem nenhum momento presencial. Acreditamos que é graças a atividades como essas que o professor pode perceber de forma direta, através de questionamentos, as dificuldades dos alunos e também dar o feedback a eles, tão importante no processo de avaliação formativa.

Já a análise dos dados do sujeito tutor do curso se baseou em dois instrumentos: o questionário aplicado aos alunos e um segundo questionário elaborado especificamente para o tutor, com questões abertas.

A utilização do mesmo questionário aplicado aos sujeitos alunos foi feita visando comparar a visão desses alunos com a visão específica do tutor que os acompanhou durante todo o curso. Analisando as respostas dadas pelo tutor, observa-se que ele assinalou um grau de concordância no conjunto das questões maior do que o dos alunos, não assinalando nenhuma questão abaixo de 4 (concordo). Isso demonstra que o tutor realmente julgou úteis as informações contidas nos relatórios de atividades SCORM.

Com relação aos elementos considerados importantes, diferentemente dos alunos, o tutor considerou o número de tentativas o mais importante, seguido das respostas do aluno e auto-avaliação do aluno. O item considerado menos relevante foi a pontuação na atividade (score). Já em relação às outras atividades do Moodle que poderiam ser utilizadas para apoiar a avaliação formativa, as atividades de chat e fórum foram os primeiros itens, seguidos das atividades de diário e livros.

Analisando as respostas dadas pelo tutor no questionário com questões abertas, podemos observar que assim como os alunos do curso, o tutor considerou que os dados registrados pelo rastreamento do SCORM podem ser utilizados para apoiar a avaliação formativa, constatando também que o conjunto de elementos do SCORM sugeridos pelo modelo é eficaz para a observação e o acompanhamento das atividades do aluno no AVA.

\section{Conclusões e Trabalhos Futuros}

A utilização de objetos de aprendizagem e a avaliação formativa são temas que têm permeado as discussões e os textos sobre EAD nos últimos anos, com diversos artigos publicados em congressos, livros e periódicos. Diversos autores há algum tempo vêm apontando as vantagens do desenvolvimento de conteúdos digitais educacionais na forma de objetos de aprendizagem. Por outro lado, a avaliação formativa tem se mostrado um importante instrumento para aproximar professores e alunos distantes geograficamente, auxiliando o acompanhamento e a regulação do processo de ensino-aprendizagem a distância.

Entretanto, a questão que nos acompanhava antes do início do trabalho era de que forma poderíamos desenvolver e encapsular objetos de aprendizagem que apoiassem o processo de avaliação formativa. Não podíamos perder de vista também que quando desenvolvemos conteúdos no formato de objetos de aprendizagem um dos principais benefícios é sua reutilização, bem como o intercâmbio com outras universidades e grupos de pesquisa.

Dessa forma, nosso objetivo foi criar um modelo agregando um conjunto de recomendações para o encapsulamento de objetos de aprendizagem, que possuíssem mecanismos para apoiar a avaliação formativa. Ao mesmo tempo, esse modelo deveria indicar como utilizar tais objetos nos diversos AVAs para a avaliação formativa, bem como orientar se eles deveriam ser licenciados e disponibilizados, assegurando sua livre utilização, distribuição e modificação por outras instituições acadêmicas. Todos esses objetivos foram atendidos nas diferentes partes desta pesquisa.

\subsection{Considerações Finais}

No que tange ao encapsulamento dos objetos, identificamos que entre os padrões existentes de portabilidade e interoperabilidade de OA, o SCORM é o modelo mais estabilizado, é o que possui os melhores mecanismos de acompanhamento do aluno, além de ser o mais utilizado e com maiores potencialidades de continuidade e manutenção pela comunidade de OA. Em decorrência do exposto, a maioria das ferramentas de autoria, mais utilizadas atualmente, publica os conteúdos no formato preconizado pelo SCORM.

Com base nessa constatação sobre o SCORM, e após um estudo aprofundado sobre o processo de avaliação formativa e sua aplicação com o apoio de tecnologias, chegamos às bases para um modelo de encapsulamento de objetos de aprendizagem que apoiassem um processo de avaliação mais formativo. Nesse modelo, identificamos claramente quais elementos do modelo de dados do SCORM deveriam ser utilizados e acrescentamos reco- 
mendações de como utilizar tais elementos para o processo de avaliação e acompanhamento do aluno. Esse modelo também contemplou as estratégias de utilização dos objetos de aprendizagem na avaliação formativa.

Não perdemos de vista nessas recomendações um de nossos objetivos de analisar licenças para objetos de aprendizagem que assegurassem sua livre utilização, distribuição e modificação. Nesse aspecto, identificamos que a licença Creative Commons é o tipo de licença mais madura e melhor redigida em termos legais, que permite que pessoas leigas facilmente a utilizem. Em nossas recomendações, não só indicamos o uso dessa licença como consideramos que para que esses objetos de aprendizagem fossem considerados abertos, eles necessariamente deveriam disponibilizar seus códigos-fonte e utilizar um modelo ou padrão de portabilidade e interoperabilidade como o SCORM.

Por ser um trabalho inovador no sentido de conjugar objetos de aprendizagem e avaliação formativa, optamos por uma pesquisa exploratória. Nosso intuito é que, a partir da presente pesquisa, novas perspectivas de pesquisa nessa área possam ser abertas.

Quanto aos resultados do estudo de caso, o que identificamos através da observação e também pelas respostas dos questionários é que, efetivamente, os dados registrados através das atividades SCORM podem ser utilizados para apoiar a avaliação formativa. Percebemos também que os sujeitos da pesquisa concordaram com o processo de avaliação formativa e com o uso do SCORM como apoio. Entre os elementos de dados do SCORM considerados mais importantes estão o número de tentativas, o estado das atividades e os comentários de auto-avaliação. Adicionalmente, verificamos que é de extrema importância o uso das ferramentas de comunicação disponíveis como forma de auxiliar a avaliação formativa.

É interessante também tecer considerações sobre todos os elementos destacados no Modelo de Encapsulamento e Utilização de Objetos de Aprendizagem Abertos.

Quanto ao encapsulamento, o que se conclui é que, apesar do SCORM estar consolidado e ser uma especificação bem detalhada, não existe ferramenta de autoria ideal para o encapsulamento, devendo ser usadas as diversas disponíveis, de acordo com a necessidade. Ademais, ainda é muito importante o uso de programação diretamente no SCO para assegurar o correto registro dos elementos do modelo de dados do SCORM, visando a avaliação formativa.

Quanto à utilização dos objetos SCORM para apoiar as tarefas do professor na avaliação formativa, verificouse que o SCORM consegue apoiar todas as quatro tarefas (desencadear, observar, comunicar e remediar) propostas por Hadji, mas que o apoio mais efetivo ocorre nas tare- fas de desencadear e observar e, em menor grau, de comunicar e remediar. Uma experiência neste sentido, envolvendo o uso de Objetos Inteligentes de Aprendizagem consonantes com o padrão SCORM foi relatada em [20] e contemplou o projeto de objetos de aprendizagem com possibilidade de adaptabilidade podendo inclusive adquirir novos conhecimentos e comportamentos no decorrer de sua existência, através da interação com os alunos e, até mesmo, com outros objetos de aprendizagem, tais como: adquirir informações sobre os alunos como as suas preferências e estilos cognitivos e verificar quais a melhores estratégias de aprendizagem em cooperação com os demais agentes.

Conclui-se que o desenvolvimento de objetos de aprendizagem SCORM (SCO) levando em consideração o registro de tais elementos de forma a serem utilizados na avaliação formativa é uma alternativa ainda pouco explorada, mas que se utilizada é uma forma eficiente e flexível de utilização da avaliação formativa sem se prender a um AVA ou mecanismo de avaliação específico.

\subsection{Perspectivas para Novas Investigações}

Este trabalho procurou abordar meios para apoiar a avaliação formativa através do encapsulamento e uso de objetos de aprendizagem abertos SCORM. Entretanto, no decorrer do desenvolvimento da pesquisa, constatamos vários desdobramentos para proceder com o trabalho de pesquisa; desdobramentos estes no aspecto metodológico, no aspecto tecnológico e também no pedagógico.

No que tange à metodologia, entendemos que seria apropriado, a partir desta pesquisa exploratória, delinear uma pesquisa descritiva, através de um estudo de caso com um experimento, caracterizando-se como uma pesquisa mista, assim como a presente pesquisa. $\mathrm{O}$ estudo de caso teria a abordagem de casos múltiplos, onde poderíamos observar os registros dos dados do SCORM, acompanhar a atividade dos tutores na avaliação formativa e ao final colher suas impressões acerca do processo. Ao mesmo tempo, poderíamos aplicar um experimento com os alunos do curso. Para tanto, seria necessária a utilização de um grupo de controle composto por uma ou duas turmas e outra turma que sofreria o experimento. Seria necessário também a aplicação de pré e pós testes aos sujeitos da pesquisa (alunos), caracterizando-se por um delineamento experimental.

Com relação ao aspecto pedagógico no projeto e desenvolvimento dos objetos de aprendizagem, entendemos que o modelo proposto não é independente da epistemologia, pois se baseia nas idéias de Coll e Zabala com uma abordagem em que o professor atua como facilitador e o aluno é um agente no processo de aprendizagem. Contudo, o modelo não possui uma delimitação epistemológica 
clara. Evidentemente, isso poderia ser uma das vantagens em se tratando de um conjunto de recomendações, mas, considerando a necessidade de interpretar e compreender um processo educativo apoiado por $\mathrm{OA}$, entendemos que o modelo epistemológico poderia ser mais bem explorado. Nesse sentido, a teoria da aprendizagem significativa de Ausubel como teoria de ensino, e a teoria sóciohistórica de Vygostsky como teoria explicativa da aprendizagem e desenvolvimento cognitivo, seriam uma das opções vislumbradas. Assim, os objetos de aprendizagem atuariam como organizadores prévios na assimilação do conhecimento, segundo a primeira teoria, ou como mediadores do processo de ensino-aprendizagem na segunda teoria. Ambas poderiam ser pontos de partida para futuros estudos, comparativos ou não.

Com base nesses estudos, poderíamos chegar a um modelo bem completo e abrangente, guiando os professores no desenvolvimento de seus objetos de aprendizagem. Isso supriria uma lacuna na metodologia de desenvolvimento de objetos de aprendizagem, lacuna essa que nos levou a usar as idéias de Coll e Zabala. Modelos como o LODAS, de Wiley, por exemplo, são bastante completos na definição de uma taxonomia, dos tipos de objetos e na forma de seqüenciar e organizar tais objetos, mas não possuem um modelo epistemológico tão bem definido e não prevêem nenhum tipo de apoio à avaliação formativa.

No aspecto tecnológico, apesar de ter sido utilizado o Moodle, o modelo é independente do AVA, pois se baseia integralmente no SCORM, sendo que qualquer outro AVA com suporte ao SCORM poderia ser utilizado. Sugerimos que nas próximas pesquisas outros AVAs sejam utilizados, tais como o Sakai, Ilias ou Dokeos. Entretanto, entendemos que o Moodle, além de sua crescente utilização no meio acadêmico, possui flexibilidade e recursos muito úteis ao desenvolvimento de cursos a distância, porém, seu módulo SCORM ainda não é estável e, em termos de avaliação formativa, são necessárias algumas melhorias, principalmente nos relatórios. Sugerimos o desenvolvimento de relatórios específicos para a avaliação formativa, permitindo a alunos e professores consultarem todos os dados registrados pelo SCORM, de forma simples e auto-explicativa, principalmente para a visualização de elementos como o 'cmi.interactions' e 'cmi.comments_from_learner', além de relatórios resumidos e comparativos. Outra funcionalidade interessante, mas que exige mudanças um pouco mais estruturais no AVA, é a possibilidade de publicação de pacotes específicos para determinados alunos ou grupos de alunos, ou uma forma de visibilidade que possa ser aplicada especificamente a um grupo de alunos.

Outro desdobramento interessante no aspecto tecnológico seria aliar as constatações deste trabalho com os estudos de objetos inteligentes de aprendizagem, pensan- do na utilização de sistemas multiagentes para a classificação, agrupamento e análise dos dados de rastreamento do SCORM registrados pelo AVA, visando acompanhar as atividades do grupo, identificando alunos com dificuldades para acionar eventos, como alertas, feedback automático para alunos e professores, seleção de novos objetos para tais alunos, entre outros.

Com relação, especificamente, ao SCORM, toda a especificação do livro de Seqüenciamento \& Navegação da versão 2004, poderia ser utilizada para o processo de avaliação formativa através de navegação não linear entre os objetos de aprendizagem, algo impossível no SCORM 1.2 que é totalmente linear. Uma sugestão de utilização dessa especificação seria o desenvolvimento de cursos inteiros em SCORM 2004, com navegação totalmente flexível entre objetos. Assim, poderíamos analisar as dificuldades dos alunos de acordo com as rotas e os objetos escolhidos nas atividades do curso SCORM.

Ao final desta pesquisa, concluímos que o desenvolvimento de objetos de aprendizagem SCORM (SCO) levando em consideração o registro de tais elementos de forma a serem utilizados na avaliação formativa é uma alternativa ainda pouco explorada, mas que se utilizada é uma forma eficiente e flexível de utilização da avaliação formativa sem se prender a um AVA ou mecanismo de avaliação específico.

\section{Referências}

[1] ADL. Advanced Distributed Learning . SCORM 2004 3rd edition. Version 1.0. 2006. http://www.adlnet.org, Fev. 2007.

[2] B. S. Bloom, J. T. Hastings, G. F. Madaus. Manual de Avaliação Formativa e Somativa do Aprendizado Escolar. Livraria Pioneira Editora, São Paulo, 1983.

[3] M. Gather Thurler. Quais as competências para operar em ciclos de aprendizagem plurianuais? Pátio, 17(2): 17-21, 2001.

[4] A. C. Gil. Como Elaborar Projetos de Pesquisa. 4 adição. Atlas, São Paulo, 2007.

[5] C. Hadji. Avaliação Desmistificada. Artmed, Porto Alegre, 2001.

[6] J. Hoffmann. Avaliação: Mito e Desafio - Uma perspectiva construtivista. Mediação, Porto Alegre, 2001.

[7] V. M. Kenski, G. P Oliveira, A. Clementino. Avaliação em movimento: estratégias formativas em cursos online. In: M. Silva, E. Santos (Org.). 
Avaliação da Aprendizagem em Educação Online. Loyola, São Paulo, 2006.

[8] C. Lankshear, M. Knobel. Pesquisa Pedagógica: Do Projeto à Implementação. Artmed, Porto Alegre, 2008.

[9] J. L Otsuka. Modelo de Suporte à Avaliação Formativa Baseado em Sistemas Multiagentes para Ambientes de EAD. Tese de doutorado. Instituto de Computação-UNICAMP, 2006.

[10] P. Perrenoud. Avaliação: Da Excelência à Regulação das Aprendizagens entre duas lógicas. Artmed, Porto Alegre, 1999.

[11] E. P. Pimentel, A. S. Alves, D. M. Oliveira, P. A. Bottaro, R. Lopes. Avaliações Adaptativas baseadas no Nível de Aquisição de Conhecimento do Aprendiz. In Anais do XVIII Simpósio Brasileiro de Informática na Educação, São Paulo, páginas 566-575, 2007.

[12] D. N. Prata. Estratégias para o Desenvolvimento de um Framework de Avaliação na Aprendizagem a Distância. In Anais do XVI Simpósio Brasileiro de Informática na Educação, Rio de Janeiro, páginas 150-159, 2003.

[13] A. P. Rodrigues. E-Avalia - Um agente para a avaliação de Ensino-Aprendizagem em Educação a Distância. Dissertação de Mestrado, Instituto de Informática-UFRGS, 2002.

[14] M. Scriven. The Methodology of Evaluation. In: Tyler, R.; Gagne, R.; Scriven, M. Perspectives of Curriculum Evaluation. American Educational Research Association, Washington, D.C., 1967.

[15] R. Weirich, I. Gasparini, R. Kemenczinski. Análise de Log para Avaliação do Comportamento do Aluno em um Ambiente Web. In Anais do XVIII Simpósio Brasileiro de Informática na Educação, São Paulo, páginas 576-586, 2007.

[16] R. K. Yin. Estudo de Caso: Planejamento e Métodos. Bookman, Porto Alegre, 2008.

[17] A. Zabala. A Prática Educativa: Como Ensinar. Tradução Ernani F. da F. Rosa. Artmed, Porto Alegre, 1998.

[18] L. A. M. Zaina. Acompanhamento do aprendizado do aluno em cursos à distância através da Web: metodologias e ferramenta. Dissertação de Mestrado, Universidade de São Paulo, Jan 2002.
[19] D. Wiley. Impediments to Learning Object Reuse and Openness as a Potential Solution. RBIE - Revista Brasileira de Informática na Educação, 17(3): 8-10, 2009.

[20] J. Silva, N. Bavaresco, R. Silveira. Projeto e desenvolvimento de um sistema multi-agentes para objetos inteligentes de aprendizagem baseado no padrão scorm. RBIE - Revista Brasileira de Informática na Educação, 16(1): 19-27, 2008.

[21] P. Long. OpenCourseWare: Simple Idea, Profound Implications. Eric Syllabus, 15(6): 12-14, 2002.

[22] L. Lessig. Free Culture: How Big Media Uses Technology and the Law to Lock Down Culture and Control Creativity. Penguin Group, New York, 2005.

[23] D. Piva Jr., M. S. Miskulin, R. L. F., C. M. Tobar. AUXILIAR: Um Sistema Inteligente para Cursos Online. RBIE - Revista Brasileira de Informática na Educação, 13(1): 52-61, 2005. 